\title{
Indications of endoscopic submucosal dissection for symptomatic benign gastrointestinal subepithelial or carcinoid tumors originating in the submucosa
}

\author{
HIDEKI KOBARA $^{1}$, HIROHITO MORI ${ }^{1}$, KAZI RAFIQ ${ }^{2}$, SHINTAROU FUJIHARA ${ }^{1}$, NORIKO NISHIYAMA ${ }^{1}$, \\ MAKI AYAKI $^{1}$, TATSUO YACHIDA ${ }^{1}$, JOHJI TANI ${ }^{1}$, HISAAKI MIYOSHI ${ }^{1}$, HIDEKI KAMADA ${ }^{1}$, \\ ASAHIRO MORISHITA ${ }^{1}$, MAKOTO ORYU ${ }^{1}$, KUNIHIKO TSUTSUI ${ }^{1}$, REIJI HABA $^{3}$ and TSUTOMU MASAKI ${ }^{1}$ \\ Departments of ${ }^{1}$ Gastroenterology and Neurology, ${ }^{2}$ Pharmacology and ${ }^{3}$ Diagnostic Pathology, \\ Faculty of Medicine, Kagawa University, Miki, Kagawa 7610793, Japan
}

Received June 7, 2013; Accepted August 19, 2013

DOI: $10.3892 / \mathrm{mco} .2013 .177$

\begin{abstract}
Endoscopic submucosal dissection (ESD) for upper gastrointestinal (GI) subepithelial tumors (SETs) originating in the muscularis propria (MP) layer is associated with numerous issues regarding secure closure and measures against accidental perforation. However, symptomatic benign GI SETs or carcinoid tumors originating in the submucosa (SM) may be safely resected en-bloc using ESD. In this study, the feasibility and safety of ESD as a novel method for endoscopic resection for such GI SETs revealed on endoscopic ultrasonography (EUS) was investigated. A total of 12 consecutive cases of patients with symptomatic benign SETs $(n=3 ; 1$ esophageal hemangioma and 2 gastric lipomas) or small carcinoid tumors $(\mathrm{n}=9 ;<10 \mathrm{~mm}$, with an extremely low risk of metastasis) originating in the SM as determined on EUS, between March, 2009 and April, 2013, were retrospectively reviewed. The lesions were resected by ESD after confirming that the tumors originated from the SM. The complication rate following en-bloc resection was also determined. En-bloc resection was achieved in all 12 cases, the mean procedure time was $45 \mathrm{~min}$ (range, 20-120 min) and no complications occurred intra- or postoperatively. There was no tumor recurrence or disease-related mortality reported during the follow-up period (median follow-up time, 13.4 months). Histopathological curative resection was achieved with ESD without complications in all 9 cases with carcinoid tumors. Therefore, if EUS reveals a SET originating in the SM without infiltration of the MP and resection is indicated due to the presence of abdominal symptoms, ESD may be a feasible option for diagnostic treatment with minimal invasiveness. However,
\end{abstract}

Correspondence to: Dr Tsutomu Masaki, Department of Gastroenterology and Neurology, Faculty of Medicine, Kagawa University, 1750-1 Ikenobe, Miki, Kita, Kagawa 7610793, Japan E-mail: tmasaki@med.kagawa-u.ac.jp

Key words: endoscopic submucosal dissection, gastrointestinal subepithelial tumors, symptomatic benign tumors, small carcinoid tumors larger-scale prospective studies are required to establish the feasibility and safety of this procedure.

\section{Introduction}

Subepithelial tumors (SETs) of the gastrointestinal tract, which may be an occasional finding on routine upper gastrointestinal (GI) endoscopy, represent a diagnostic and therapeutic challenge. SETs include malignant tumors, such as GI stromal tumors, malignant lymphomas, carcinoid tumors and gastric cancers resembling SETs, as well as benign tumors, such as leiomyomas, aberrant pancreas and lipomas. The most common type of tumor is gastrointestinal stromal tumor (GIST), first described by Mazur and Clark (1). GISTs originate from the submucosa (SM) and muscularis propria (MP) of the GI tract. En-bloc resection is widely performed for the treatment of definite GISTs. Currently, minimally invasive local resection techniques, such as hybrid natural orifice transluminal endoscopic surgery (NOTES), which consists of endoscopic full-thickness resection (EFTR), have been developed for the treatment of endoluminal GISTs $(2,3)$. Furthermore, endoscopic diagnostic methods for SETs have been proposed $(4,5)$, including our safe and reliable method of bloc biopsy performed under a direct endoscopic view by using submucosal endoscopy with a mucosal flap method (SEMF) (4). Although considered by some as a novel optimal endoscopic mini-invasive technique for the management of SETs, there are currently no established guidelines for endoscopic submucosal dissection (ESD). ESD possesses several advantages over conventional endoscopic mucosal resection, including a higher en-bloc resection rate and more accurate pathological estimation. It is currently gaining broad acceptance for the treatment of early neoplastic lesions and was also proposed for the treatment of SETs (6-9). Recently, studies on ESD for SETs originating in the MP layer of the GI wall reported some limitations with respect to complete resection and safety, which may be attributed to difficulties with the endoscopic platform and a high perforation rate $(8,10,11)$. Thus, the indications and utility of ESD for SETs originating within the SM remain undetermined. There have been a number of studies on the successful treatment of symptomatic SETs 
originating in the SM and carcinoid tumors (12-15). Endoscopic ultrasonography (EUS) is considered to be the most accurate method for determining the size, layer of origin, margins and echogenicity of SETs. As it is technically possible to perform ESD for the en-bloc resection of SETs revealed by EUS as a remnant in the SM layer, we recommend ESD as a suitable treatment for symptomatic benign GI SETs and small carcinoid tumors originating in the SM. In this study, we aimed to present the efficacy and safety of ESD treatment in 12 patients with SETs originating in the SM layer and discuss the predictors of treatment success.

\section{Patients and methods}

Selection criteria. This retrospective non-comparative study of a case series reviewed the cases of 12 consecutive patients who underwent ESD for SETs at Kagawa University Hospital (Kagawa, Japan) between March, 2009 and April, 2013 and met the following selection criteria: lesion contained within the SM layer; symptomatic SET strongly suspected to be a benign tumor on conventional endoscopic imaging, EUS and computed tomography (CT); confirmed histological diagnosis of carcinoid tumor; carcinoid tumor $\leq 10 \mathrm{~mm}$; no lymph node or distant metastasis found prior to ESD. Ethical approval was granted from the Ethics Committee of Kagawa University (Kagawa, Japan). Prior written patient informed consent was obtained.

Patients. The patients (6 males and 6 females) had a mean age of 60.4 years (range, $41-78$ years) and had symptomatic benign SETs $(n=3 ; 1$ had an esophageal hemangioma and 2 had gastric lipomas) or small carcinoid tumors $(n=9)$ (Table I). In the 3 symptomatic SET cases, tissue sampling was not performed prior to ESD. All the regions were evaluated by routine EUS (high-frequency miniprobe, $20 \mathrm{MHz}, \mathrm{UM}-3 \mathrm{R}$; Olympus Medical Systems, Tokyo, Japan) and CT. In all the cases, the EUS revealed a mass localized in the SM, which was confirmed on CT not to invade the surrounding organs.

ESD. ESD was performed with the use of a single-channel scope (GIF-H260Z; Olympus Medical Systems) and an electrosurgical unit (VIO300D; ERBE Elektromedizin GmbH, Tübingen, Germany). A short transparent cap was attached to the tip of endoscope to provide a constant endoscopic view and to apply tension to the connective tissue for submucosal dissection. ESD was mainly performed as follows: First, marker dots were placed $\sim 5 \mathrm{~mm}$ from the lesion and, using a 23-gauge disposable needle, a submucosal injection of several milliliters of $0.4 \%$ hyaluronate sodium solution (MucoUp; Johnson \& Johnson K.K., Tokyo, Japan) was performed around the lesion to lift it off the MP layer. The mucosa was then incised outside the marker dots, using a needle knife (KD-650L or 650U; Olympus Medical Systems). The submucosal connective tissue beneath the lesion was gradually dissected in the SM layer directly above the muscular coat with the needle knife or an improved insulated-tip (IT-2) knife (KD-611L; Olympus Medical Systems). The solution was injected repeatedly during the dissection if required. Finally, the lesion was completely resected from the MP layer with the needle or the IT-2 knife. Hemostasis of the large blood vessels running through the layers prior to dissection prevented intraoperative bleeding. All patients were administered intravenous midazolam $(0.05 \mathrm{mg} / \mathrm{kg})$ and pethidine $(50 \mathrm{mg})$ prior to the procedure. All the procedures were performed by an experienced endoscopist (H.K.: >200 successfully performed ESD procedures). During the entire procedure bleeding was controlled using hemostatic forceps (FD-410LR; Olympus Medical Systems). The patients were allowed to intake a small amount of water immediately after the treatment. If there were no complications, the patients were permitted to intake soft food on the following day and were discharged within 1 week.

The en-bloc resection rate, procedure time, complications and residual local recurrence of the resected lesion were evaluated. In addition, for the 9 small carcinoid tumors, complete resection was histologically evaluated. An en-bloc resection was defined as an one-piece resection including tumor enucleation. The procedure time was measured from the time of marker dot placement to the resection of the lesion. The complications included the incidence of bleeding requiring blood transfusion, perforation and intraluminal stenosis requiring additional surgery. For the 9 small carcinoid tumors, incomplete resection was defined as the extension of the tumor into the lateral or vertical resection margin.

Follow-up. All patients were scheduled for follow-up by standard endoscopy and EUS every 3 months within the first year following treatment to confirm the healing of the artificial ulcer and assess any residual tumor. Thereafter, follow-up continued on an annual basis to monitor for local recurrence and other lesions. Biopsy specimens were collected from any ulcerative lesion identified during follow-up to histologically confirm the presence of residual tumor and local recurrence. Abdominal and pelvic CT was performed to assess the pararectal lymph nodes and identify distant metastasis as deemed necessary. Three cases (1,2 and 6) are presented subsequently, cases 1 and 2 are rare, and therefore of interest and case 6 is typical of cartinoid cases.

Case 1. A 41-year-old female presented with dysphagia. GI endoscopy revealed a bluish submucosal mass (diameter, $25 \mathrm{~mm}$ ) in the upper esophagus. The EUS revealed a hypoechoic mass localized in the SM layer. The mass was diagnosed as a submucosal hemangioma of the esophagus. ESD was performed under general anesthesia after obtaining the patient's informed consent. Although a submucosal vascular plexus was identified, loose connective tissue was present in the SM directly above the muscular coat, enabling the dissection of the target layer. There were several large blood vessels running through the muscular coat; however, secure hemostasis with a coagulation forceps blocked the flow in these vessels. The clear operative field for ESD permitted the careful dissection required to avoid rupturing the hemangioma. The resected en-bloc specimen included a dark purple mass. The histopathological results revealed the outgrowth of dilated blood vessels surrounded by flat endothelial cells in the $\mathrm{SM}$, leading to the diagnosis of cavernous hemangioma. The patient has been recurrence-free for 6 months (17).

Case 2. In this case, ESD was selected as diagnostic treatment for a symptomatic gastric SET originating in the 
Table I. Clinicopathological data of patients with subepithelial tumors $(n=12)$.

\begin{tabular}{cccccccc}
\hline Case & Age (years)/gender & Location & Symptoms & Tumor size $(\mathrm{mm})$ & Layer & Echoic & Pathology \\
\hline 1 & $41 / \mathrm{F}$ & Esophagus/cervical & Yes & 25 & SM & Hypo & Hemangioma \\
2 & $76 / \mathrm{F}$ & Stomach/antrum & Yes & 30 & SM & Hyper & Lipoma \\
3 & $41 / \mathrm{F}$ & Stomach/antrum & Yes & 20 & SM & Hyper & Lipoma \\
4 & $64 / \mathrm{M}$ & Stomach/body & No & 9 & SM & Hypo & WDNET \\
5 & $78 / \mathrm{F}$ & Duodenum/bulb & No & 8 & SM & Hypo & WDNET \\
6 & $51 / \mathrm{M}$ & Rectum/Rb & No & 9 & SM & Hypo & WDNET \\
7 & $63 / \mathrm{M}$ & Rectum/Rb & No & 7 & SM & Hypo & WDNET \\
8 & $69 / \mathrm{M}$ & Rectum/Ra & No & 8 & SM & Hypo & WDNET \\
9 & $60 / \mathrm{F}$ & Rectum/Rb & No & 10 & SM & Hypo & WDNET \\
10 & $54 / \mathrm{F}$ & Rectum/Rb & No & 4 & SM & Hypo & WDNET \\
11 & $64 / \mathrm{M}$ & Rectum/Rb & No & 2 & SM & Hypo & WDNET \\
12 & $64 / \mathrm{M}$ & Rectum/Ra & No & 5 & SM & Hypo & WDNET \\
\hline
\end{tabular}

F, female; M, male; SM, submucosa; WDNET, well-differentiated neuroendocrine tumor; Rb, below the rectum; Ra, above the rectum; yrs, years.

submucosal layer, which was ultimately proven to be a lipoma. A 75-year-old female presented with a sensation of abdominal fullness brought on by a GI obstruction by a SET (diameter, $30 \mathrm{~mm}$ ) in the prepyloric area (Fig. 1A), causing ball-valve syndrome. The EUS revealed a hyperechoic mass localized in the SM (Fig. 1B), although it could not be accurately diagnosed with EUS-fine needle aspiration (FNA). Although we recommended obtaining a tissue sample using our method of bloc biopsy with SEMF (4), the patient opted for endoscopic resection of the SET to relieve the abdominal fullness. After obtaining informed consent, we selected tumor enucleation as the minimum resection, using ESD to prevent postoperative prepyloric stenosis. The yellowish tumor identified under a direct endoscopic view during ESD was suggestive of a lipoma. The submucosa was easily dissected without the need for any specific technique (Fig. 1C) and en-bloc resection was achieved in $41 \mathrm{~min}$. The size of the tumor was $30 \times 20 \mathrm{~mm}$ (Fig. 1D). There were no complications and the sensation of abdominal fullness disappeared immediately after ESD. The follow-up endoscopy 2 months following ESD revealed no residual tumor or gastrointestinal obstruction (Fig. 1E). The histopathological examination confirmed the diagnosis of a lipoma.

Case 6. A 51-year-old man was diagnosed with a rectal carcinoid tumor (diameter, $9 \mathrm{~mm}$ ) located below the rectal area (Fig. 2A). The EUS revealed a hypoechoic mass originating in the SM layer, with no invasion of the MP layer (Fig. 2B). ESD was performed using a needle knife (KD-650U; Olympus Medical Systems) after obtaining the patient's informed consent. The SM was dissected immediately above the MP layer to avoid a positive vertical resection margin (Fig.2C). En-bloc resection was successfully completed in $52 \mathrm{~min}$, without any complications (Fig. 2D). The histological examination confirmed the diagnosis of a rectal carcinoid tumor, classified as a well-differentiated neuroendocrine tumor, and a negative resection margin. The patient has been recurrence-free for 6 months.

\section{Results}

Clinical outcome and complications. The clinical outcomes according to the endoscopic treatment modality are summarized in Table II. The entire procedure was successfully completed in all the patients. En-bloc resection was performed in all 12 cases and the mean procedure time was 45 min (range, 20-120 $\mathrm{min}$ ). There were no reported complications during or after the procedure and there were no cases of intraluminal stenosis requiring additional surgery.

Follow-up and histopathology. The median follow-up was 13.4 months (range, 1-39 months) and there was no reported recurrence or disease-related mortality during the follow-up period. Histopathologically, curative resection was achieved in all 9 carcinoid tumor cases, which were all classified as well-differentiated neuroendocrine tumors (proliferation index $<2 \%$ ).

\section{Discussion}

There is currently no consensus on the optimal strategy for the endoscopic treatment of SETs. Endoscopic submucosal resection (ESMR) has been reported to be effective for the treatment of SETs (12-15) and it is usually reserved for lesions that are confined to the submucosal or mucosal layers, due to the increased risk of perforation associated with ESMR of lesions originating in the MP layer. However, ESMR occasionally requires a large en-bloc resection and secure hemostasis may prove challenging. Recently, ESD, which was developed from the endoscopic mucosal resection method, was introduced as a novel method of endoscopic treatment that allows for such resection and hemostatic management, as well as precise histological staging. ESD may also be more effective in preventing disease recurrence compared to the conventional ESMR. Indeed, there are already available studies on the successful application of the ESD procedure for the diagnostic treatment of several types of GI SETs, such as lipomas and carcinoids (16-19). 

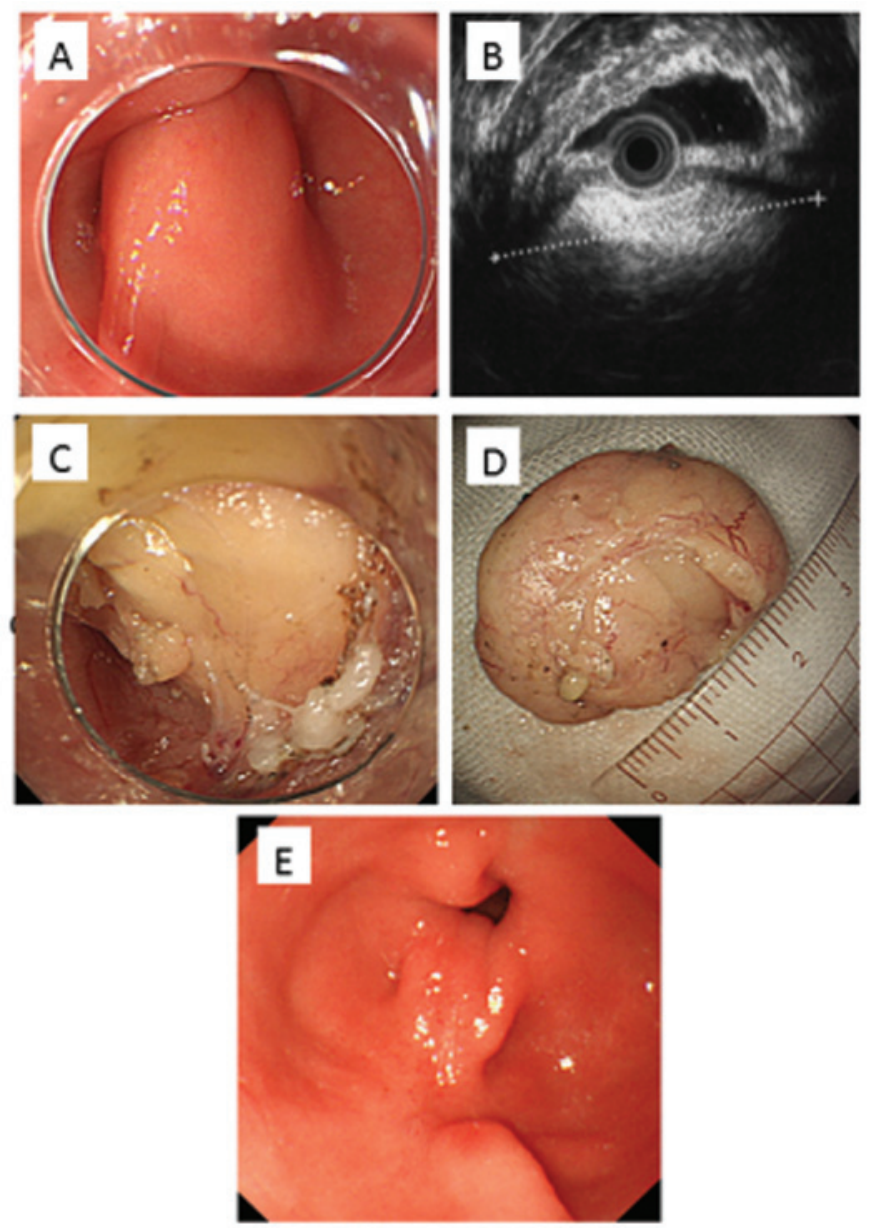

Figure 1. Case 2. (A) Endoscopic view of the prepyloric area revealed a subepithelial tumor causing ball-valve syndrome. (B) Endoscopic ultrasonography revealed a hyperechoic mass localized in the submucosa. (C) A yellowish lipoma in the submucosal layer that was resected en-bloc by endoscopic submucosal dissection (ESD) under direct vision. (D) Macroscopic image of the resected specimen (30x20 mm). The histopathological examination confirmed the diagnosis of gastric lipoma. (E) Follow-up endoscopy 2 months after the ESD revealed no tumor or gastrointestinal obstruction.
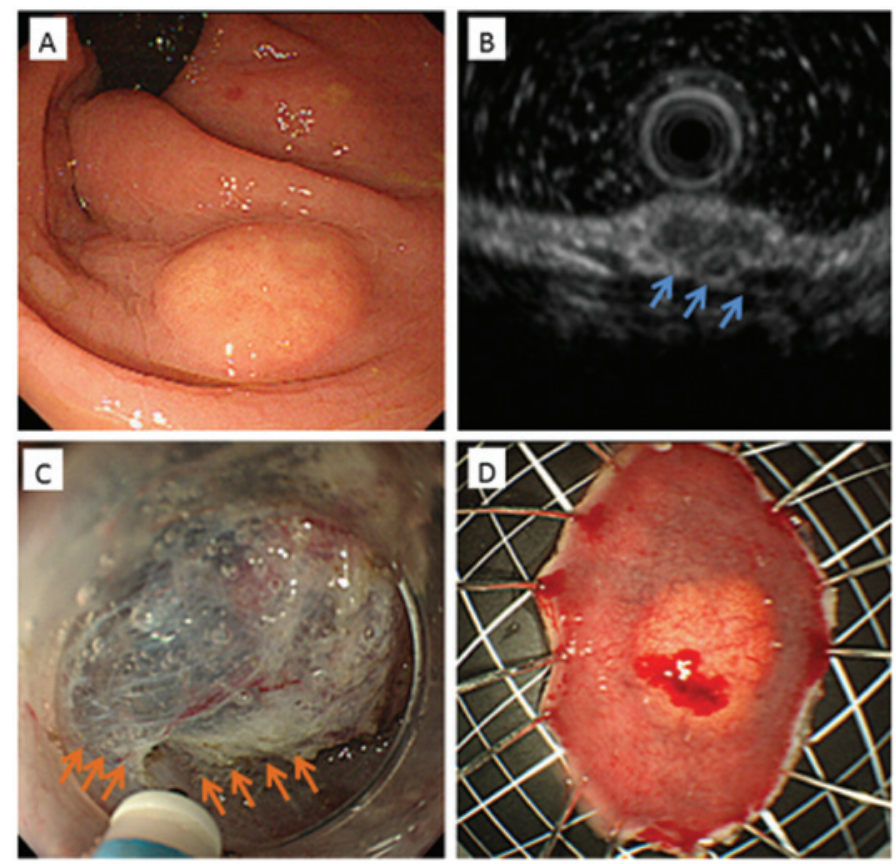

Figure 2. Case 6. (A) Endoscopic view below the rectum revealed a carcinoid tumor (diameter, $9 \mathrm{~mm}$ ). (B) Endoscopic ultrasonography revealed a hypoechoic mass originating in the submucosal layer (blue arrows), without invasion of the muscularis propria (MP). (C) The submucosa immediately above the MP (orange arrows) was dissected to avoid a positive vertical tumor margin. (D) Macroscopic image of the specimen (22x20 mm) removed by en-bloc endoscopic submucosal dissection. The histopathological examination confirmed the diagnosis of rectal carcinoid classified as a well-differentiated neuroendocrine tumor. 
Table II. Clinical outcomes according to the endoscopic treatment modality $(n=12)$.

\begin{tabular}{rccccccc}
\hline Case & Pathology & $\begin{array}{c}\text { Procedure } \\
\text { time (min) }\end{array}$ & $\begin{array}{c}\text { Complete } \\
\text { resection }\end{array}$ & Complications & $\begin{array}{c}\text { Follow-up } \\
\text { period (months) }\end{array}$ & Recurrence & $\begin{array}{c}\text { Additional } \\
\text { surgery }\end{array}$ \\
\hline 1 & Hemangioma & 120 & Yes & No & 6 & No & No \\
2 & Lipoma & 41 & Yes & No & 4 & No & No \\
3 & Lipoma & 32 & Yes & No & 18 & No & No \\
4 & WDNET & 37 & Yes & No & 36 & No & No \\
5 & WDNET & 63 & Yes & No & 2 & No & No \\
6 & WDNET & 52 & Yes & No & 4 & No & No \\
7 & WDNET & 30 & Yes & No & 13 & No & No \\
8 & WDNET & 49 & Yes & No & 35 & No & No \\
9 & WDNET & 37 & Yes & No & 39 & No & No \\
10 & WDNET & 28 & Yes & No & 2 & No & No \\
11 & WDNET & 31 & Yes & No & 1 & No & No \\
12 & WDNET & 20 & Yes & No & 1 & & \\
\hline
\end{tabular}

WDNET, well-differentiated neuroendocrine tumor.

We recommend ESD as a suitable treatment for symptomatic SET with gastrointestinal obstruction. In the present study, the treatment of symptomatic benign SETs (1 esophageal hemangioma and 2 gastric lipomas) was successful and the symptoms were eliminated. Moreover, we previously reported the first case of a submucosal esophageal hemangioma successfully removed en-bloc by ESD (17). Since conventional endoscopic therapy, such as ESMR, has been associated with the risk of bleeding and recurrence of hemangiomas (20), en-bloc removal by ESD may prove to be a viable treatment option in these cases. Furthermore, ESD treatment may be indicated if EUS and CT reveal that the tumors are confined to the SM layer, without large inflow vessels. In addition, radical ESD treatment was possible in 2 cases of symptomatic gastric lipomas in the present study. In case 2, considering the issue of postoperative pyloric stenosis, we performed tumor enucleation with minimum resection using ESD. Endoscopic observation at 3 months postoperatively revealed healing of the surgical site with scar formation, but without deformation or stenosis. This suggests that, if preoperative diagnostic imaging reveals a typical lipoma, tumor enucleation that takes into account postoperative stenosis may be an effective treatment option.

ESD may be the optimal treatment method for symptomatic SETs originating in the SM layer, since it allows for secure hemostasis and en-bloc resection under direct vision. Indeed, the efficacy of endoscopic treatment using ESD for SETs, mainly GISTs, originating in the MP layer of the GI tract was previously reported $(8,10,11)$. However, since GI full-thickness layers must be resected to treat SETs in the MP layer, the site must be securely closed and the vasculature in all the layers must be carefully managed. There is also a limit to the en-bloc resection that may be performed without damaging the tumor in a narrow operative field accessible through a small opening. Three case series with similar inclusion criteria and methods reported comparable rates of successful en-bloc resection for SETs originating in the MP layer (61-68\%) and severe complications due to perforation $(5.4,0$ and $12 \%$, respectively) $(8,21,22)$. Therefore, minimally invasive local resection techniques, such as NOTES, appear to be suitable for the treatment of SETs originating from the MP layer $(2,3)$.

We recommend the algorithm presented in Fig. 3 for the management of indefinite SETs, except carcinoid tumors. As was demonstrated by the present study, ESD may be the treatment of choice for symptomatic SETs or SETs increasing in size, when found to be originating in the SM layer on EUS. For asymptomatic SETs and SETs stable in size originating in the SM layer (excluding lipomas, vascular lesions or cysts identified on EUS and CT), tissue sampling methods, such as EUS-FNA or bloc biopsy with SEMF, are recommended. According to the histological findings, surveillance or ESD treatment may be selected. If a SET originating in the MP layer is identified on EUS, tissue sampling is recommended to distinguish GISTs from other benign tumors (e.g., leiomyoma and schwannoma). Minimally invasive local resection, such as EFTR, is recommended if the lesion is confirmed to be a GIST. If tissue sampling methods reveal a benign SET, surveillance over the short term is not required.

As regards the possibility of ESD treatment for carcinoid tumors, tumor size is considered to be the most important factor associated with the metastasis of rectal carcinoid tumors $(23,24)$. Endoscopic treatment is considered to be curative for small carcinoid tumors $(<10 \mathrm{~mm})$ with an extremely low risk of metastasis $(23,24)$. However, there is still some controversy over the optimal endoscopic method for the resection of rectal carcinoid tumors. Endoscopic treatment for carcinoid tumors requires special techniques for deeper resection to achieve clear margins, since $\sim 75 \%$ of the tumors extend into the SM layer (25). Conventional ESMR was found to allow for a lower rate of en-bloc resection, particularly with respect to the vertical margin (26); therefore, to improve resectability, an ESMR technique utilizing a ligation device was designed and found to achieve a significantly deeper vertical resection margin and a higher curative resection rate (15). More recently, ESD was reported to be an effective method for the treatment of rectal carcinoid tumors $(18,19)$. 


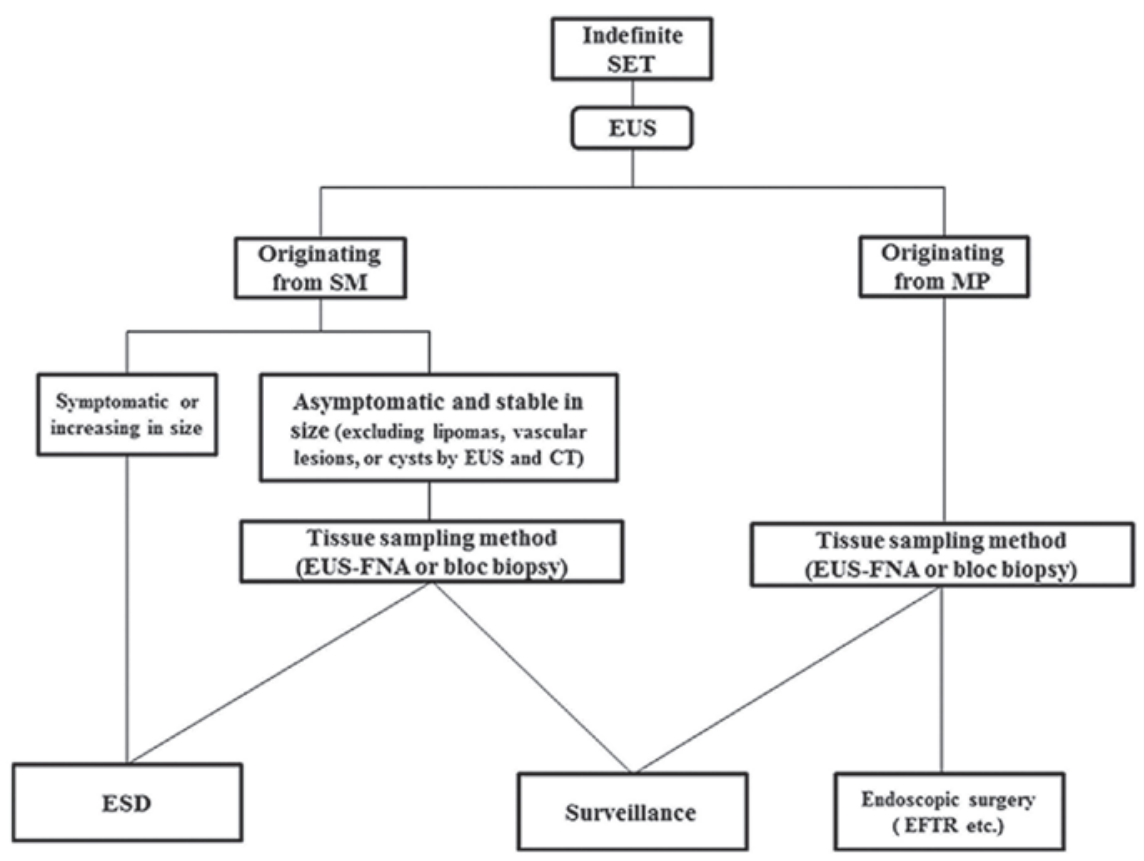

Figure 3. Proposed treatment algorithm for gastrointestinal subepithelial tumor (SET). EUS, endoscopic ultrasonography; SM, submucosa; MP, muscularis propria; CT, computed tomography; FNA, fine needle aspiration; ESD, endoscopic submucosal dissection; EFTR, endoscopic full-thickness resection.

The advantages of ESD over previously used treatment methods for carcinoids are significant. Firstly, ESD achieves clear vertical resection margins by dissecting the SM immediately above the MP layer. Secondly, ESD enables precise histological assessment of the resected specimen. For these reasons, we recommend ESD as the most suitable method for the treatment of carcinoid tumors of the SM. In our study, histopathological curative resection by ESD was achieved without complications in all 9 cases of carcinoid tumors, although the mean procedure time was longer $(38.6 \mathrm{~min})$ compared to that reported by previous studies $(18,19,26)$. This may be attributed to the longer time required to achieve a clear vertical margin while avoiding perforation; however, this also resulted in the absence of any complications. However, ESD may involve certain risks due to its technical difficulties; in addition, it may be more time-consuming. Therefore, only experienced endoscopists should perform ESD treatment for carcinoids. In this study, none of the cases exhibited tumor recurrence during follow-up. We suggest the algorithm presented in Fig. 4 for the management of definite carcinoids. Diagnostic ESD is recommended for small carcinoid tumors $(<10 \mathrm{~mm})$ when EUS reveals tumor invasion within the SM. If histology indicates that ESD was not curative, additional surgery is required. Conventional surgery is recommended for carcinoid tumors $>10 \mathrm{~mm}$ in size or when EUS reveals tumor invasion of the MP layer. In the case of rectal carcinoid tumors, however, those sized $<10 \mathrm{~mm}$ were reported to result in $<2 \%$ chance of metastasis (27). Careful follow-up is required to detect any local recurrence, as complete removal may be possible in repeat procedures. In carcinoid tumors sized $<10 \mathrm{~mm}$, further prospective studies are required to determine the feasibility of ESD, the long-term recurrence rates and patient survival. The advantages of the present study are that it suggests novel indications and the validity of

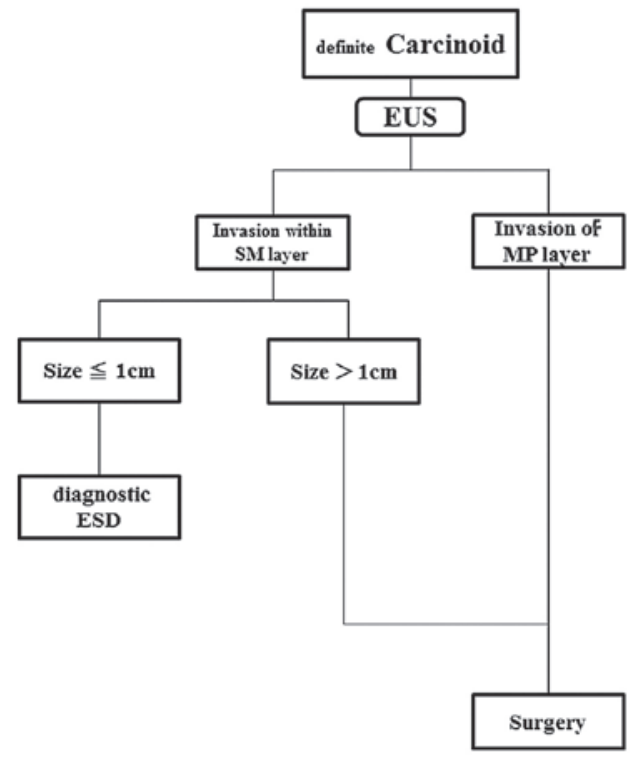

Figure 4. Flowchart of treatment strategy for carcinoid tumors based on recent developments. EUS, endoscopic ultrasonography; SM, submucosa; MP, muscularis propria; ESD, endoscopic submucosal dissection.

ESD treatment for symptomatic SETs and carcinoid tumors $<10 \mathrm{~mm}$ in size that originate in the SM, as well as the inclusion of a novel strategy based on flowcharts for SETs and carcinoid tumors. However, the limitation of the present study was that it was a single-center, non-comparative study with a small sample size. Therefore, larger scale prospective studies are required to verify our findings.

In conclusion, if EUS reveals a SET originating in the SM layer, without infiltration of the MP layer, and resection is required to alleviate abdominal symptoms, the minimally 
invasive ESD procedure may be considered as a feasible diagnostic treatment option.

\section{References}

1. Mazur MT and Clark HB: Gastric stromal tumors. Reappraisal of histogenesis. Am J Surg Pathol 7: 507-519, 1983.

2. Mori H, Kobara H, Kobayashi M, et al: Establishment of pure NOTES procedure using a conventional flexible endoscope: review of six cases of gastric gastrointestinal stromal tumor. Endoscopy 43: 631-634, 2011.

3. von Renteln D, Rosch T, Kratt T, et al: Endoscopic full-thickness resection of submucosal gastric tumors. Dig Dis Sci 57 1298-1303, 2012.

4. Kobara H, Mori H, Fujihara S, et al: Bloc biopsy by using submucosal endoscopy with a mucosal flap method for gastric subepithelial tumor tissue sampling (with video). Gastrointest Endosc 77: 141-145, 2013.

5. de la Serna-Higuera C, Perez-Miranda M, Diez-Redondo P, et al: EUS-guided single-incision needle-knife biopsy: description and results of a new method for tissue sampling of subepithelial GI tumors (with video). Gastrointest Endosc 74: 672-676, 2011.

6. Ono H, Kondo H, Gotoda T, et al: Endoscopic mucosal resection for treatment of early gastric cancer. Gut 48: 225-229, 2001.

7. Katoh T, Itoh Y, Mohri T, et al: Endoscopic enucleation of gastrointestinal stromal tumors of the stomach: report of five cases. World J Gastroenterol 14: 2609-2611, 2008.

8. Bialek A, Wiechowska-Kozlowska A, Pertklewicz J, et al: Endoscopic submucosal dissection for treatment of gastric subepithelial tumors (with video). Gastrointest Endosc 75: 276-286, 2012.

9. Rosch T, Sarbia M, Schumacher B, et al: Attempted endoscopic en bloc resection of mucosal and submucosal tumors using insulated-tip knives: a pilot series. Endoscopy 36: 788-801, 2004

10. Bialek A, Wiechowska-Kozlowska A and Huk J: Endoscopic submucosal dissection of large gastric stromal tumor arising from muscularis propria. Clin Gastroenterol Hepatol 8: e119-e120, 2010

11. Inoue H, Ikeda H, Hosoya T, et al: Submucosal endoscopic tumor resection for subepithelial tumors in the esophagus and cardia. Endoscopy 44: 225-230, 2012.

12. Hyun JH, Jeen YT, Chun HJ, et al: Endoscopic resection of submucosal tumor of the esophagus: results in 62 patients. Endoscopy 29: 165-170, 1997.

13. Kojima T, Takahashi H, Parra-Blanco A, et al: Diagnosis of submucosal tumor of the upper GI tract by endoscopic resection. Gastrointest Endosc 50: 516-522, 1999.
14. Waxman I, Saitoh Y, Raju GS, et al: High-frequency probe EUS-assisted endoscopic mucosal resection: a therapeutic strategy for submucosal tumors of the GI tract. Gastrointest Endosc 55: 44-49, 2002.

15. Ono A, Fujii T, Saito Y, et al: Endoscopic submucosal resection of rectal carcinoid tumors with a ligation device. Gastrointest Endosc 57: 583-587, 2003.

16. Yoshida T, Fujisaki J, Suganuma T, et al: Successful en bloc resection of a $5 \mathrm{~cm}$ symptomatic sessile gastric lipoma by endoscopic submucosal dissection. Dig Endosc 24: 282, 2012.

17. Kobara H, Mori H and Masaki T: Successful en bloc resection of an esophageal hemangioma by endoscopic submucosal dissection. Endoscopy 44 (Suppl 2): E134-E135, 2012.

18. Lee DS, Jeon SW, Park SY, et al: The feasibility of endoscopic submucosal dissection for rectal carcinoid tumors: comparison with endoscopic mucosal resection. Endoscopy 42: 647-651, 2010.

19. Park HW, Byeon JS, Park YS, et al: Endoscopic submucosal dissection for treatment of rectal carcinoid tumors. Gastrointest Endosc 72: 143-149, 2010.

20. Urakami T, Kondo K, Kasugai T, et al: A case of recurrent esophageal cavernous hemangioma increasing rapidly after surgery. Jpn J Thorac Cardiovasc Surg 46: 1206-1210, 1998 (In Japanese).

21. Lee IL, Lin PY, Tung SY, et al: Endoscopic submucosal dissection for the treatment of intraluminal gastric subepithelial tumors originating from the muscularis propria layer. Endoscopy 38 : 1024-1028, 2006.

22. Hwang JC, Kim JH, Kim JH, et al: Endoscopic resection for the treatment of gastric subepithelial tumors originated from the muscularis propria layer. Hepatogastroenterology 56: 1281-1286, 2009.

23. Park $\mathrm{CH}$, Cheon JH, Kim JO, et al: Criteria for decision making after endoscopic resection of well-differentiated rectal carcinoids with regard to potential lymphatic spread. Endoscopy 43: 790-795, 2011.

24. Soga J: Carcinoids of the rectum: an evaluation of 1,271 reported cases. Surg Today 27: 112-119, 1997.

25. Matsumoto T, Iida M, Suekane H, et al: Endoscopic ultrasonography in rectal carcinoid tumors: contribution to selection of therapy. Gastrointest Endosc 37: 539-542, 1991.

26. Zhou PH, Yao LQ, Qin XY, et al: Advantages of endoscopic submucosal dissection with needle-knife over endoscopic mucosal resection for small rectal carcinoid tumors: a retrospective study. Surg Endosc 24: 2607-2612, 2010.

27. Modlin IM, Oberg K, Chung DC, et al: Gastroenteropancreatic neuroendocrine tumours. Lancet Oncol 9: 61-72, 2008. 